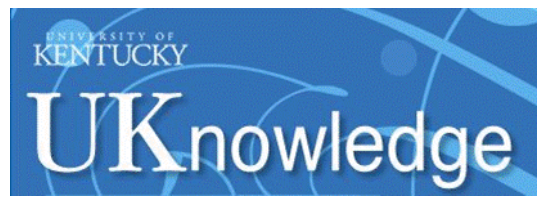

University of Kentucky

UKnowledge

Biosystems and Agricultural Engineering Faculty Publications

Biosystems and Agricultural Engineering

2015

\title{
An Optical Sprayer Nozzle Flow Rate Sensor
}

Joseph S. Dvorak

University of Kentucky, joe.dvorak@uky.edu

Luke E. Bryant

University of Kentucky

Follow this and additional works at: https://uknowledge.uky.edu/bae_facpub

Part of the Agriculture Commons, Bioresource and Agricultural Engineering Commons, and the Optics Commons

Right click to open a feedback form in a new tab to let us know how this document benefits you.

\section{Repository Citation}

Dvorak, Joseph S. and Bryant, Luke E., "An Optical Sprayer Nozzle Flow Rate Sensor" (2015). Biosystems and Agricultural Engineering Faculty Publications. 46.

https://uknowledge.uky.edu/bae_facpub/46

This Article is brought to you for free and open access by the Biosystems and Agricultural Engineering at UKnowledge. It has been accepted for inclusion in Biosystems and Agricultural Engineering Faculty Publications by an authorized administrator of UKnowledge. For more information, please contact UKnowledge@lsv.uky.edu. 


\section{An Optical Sprayer Nozzle Flow Rate Sensor}

Digital Object Identifier (DOI)

https://doi.org/10.13031/trans.58.10765

\section{Notes/Citation Information}

Published in Transactions of the ASABE, v. 58, issue 2, p. 251-259.

(c) 2015 American Society of Agricultural and Biological Engineers

The copyright holder has granted the permission for posting the article here. 


\title{
AN OpticAl SpRAYER NOZZLE Flow RATE SENSOR
}

\author{
J. S. Dvorak, L. E. Bryant
}

\begin{abstract}
Ensuring proper flow rates from each nozzle on an agricultural sprayer has become even more important as advances continue to be made in precision application technology. In this article, we describe the structure and testing of a sensor technology based on optical cross-correlation to determine the flow rate of individual sprayer nozzles. An advantage of this technology is that it does not require that impellers or other components be placed in the flow, which could cause plugging. The only moving part in the entire system is a solenoid used to inject a tracer dye. The objective of this study was to evaluate the ability of this sensor technology to determine volumetric flow rate from a single nozzle as used on an agricultural sprayer system. Tests were conducted at four system pressures $(100,200,300$, and $400 \mathrm{kPa})$ and with four nozzles in the $80^{\circ}$ extended-range flat spray nozzle family to produce different flow rates (from 0.46 to $2.74 \mathrm{~L} \mathrm{~min}^{-1}$ ). Thirty-five samples were taken for each test condition. Five randomly selected samples were used to create a calibration curve for the sensor system, and the remaining 30 samples were used for validation of performance. The worst absolute error for flow rate estimation in percent was $7.9 \%$, while the mean absolute error in percent was $1.6 \%$ for all measurements. While the flow rate estimates for the XRC8006 nozzle at $100 \mathrm{kPa}$ exhibited bias in the errors, for the rest of the test conditions, the errors were clustered around zero. The overall mean absolute error of $1.6 \%$ indicates the capability of this sensor technology to monitor flow rate of individual nozzles. However, the bias in errors in one test condition demonstrate that more testing needs to be conducted with a variety of different nozzle types and sprayer configurations before this sensor technology can be considered applicable for all sprayer applications.
\end{abstract}

Keywords. Cross-correlation, Flow rate, Nozzle, Optical, Sensor, Sprayer.

$\mathrm{A}$ gricultural sprayers rely on the application of proper amounts of chemicals to achieve the intended agronomic goals. Application of incorrect levels of chemicals can result in a waste of money and resources (Grisso et al., 1989), environmental issues (Varner et al., 1990), and reduced yields (Wesley et al., 2013). Improvements such as variable rate control, boom section control, and turn-row compensation aim to reduce the improper application of chemicals. Unfortunately, these improvements can introduce further complications. Sharda et al. (2010) monitored the pressure at individual nozzles on a sprayer boom when using section control and found pressure spikes as high as 35.7\% above normal. This increased pressure caused variations in flow, assuming that the nozzle geometry remained constant during these spikes. Other flow variations can occur at constant pressures as the nozzle geometry changes. Many researchers (Hofman and Solseng, 1986; Menzies et al., 1976; Ozkan et al., 1992; Reichard et al., 1991) have documented the effect of nozzle wear on flow rates. Field surveys of golf course pesticide applicators in Nebraska by Varner et al. (1990) found that just over $25 \%$ of applicators

Submitted for review in May 2014 as manuscript number MS 10765; approved for publication by the Machinery Systems Community of ASABE in January 2015.

The authors are Joseph S. Dvorak, ASABE Member, Assistant Professor, and Luke E. Bryant, Undergraduate Student, Department of Biosystems and Agricultural Engineering, University of Kentucky, Lexington, Kentucky. Corresponding author: Joseph S. Dvorak, 128 C.E. Barnhart Building, University of Kentucky, Lexington, KY 405460276; phone: 859-257-5658; e-mail: joe.dvorak@uky.edu. were not operating properly maintained sprayers, and the sprayers had coefficients of variation between the nozzles of over $10 \%$. Nozzles can also be partially plugged, which results in geometry and flow changes at constant pressure for as long as the obstruction is present. Wesley et al. (2013) found that variations in solenoids can cause small but consistent application rate errors in variable-rate systems. Even brand new nozzles can exhibit flow rate variations, as Huyghebaert et al. (2001) found that 20\% of new nozzles exceeded manufacturing tolerances on average.

Based on the need to monitor for flow rate variations at individual nozzles, several companies have come out with systems to do just that. Their offerings range from electronic systems with alarms (Teejet, Wheaton, Ill.) to simple rotameter designs that must be inspected visually by the operator (Wilger, Saskatoon, Saskatchewan, Canada). However, these current systems have several significant limitations. First, these systems are all based on elements that must be placed in the flow. These components can jam, wear, or be damaged, causing incorrect flow measurements or even impeding the flow itself. Extra moving parts for each nozzle also produce reliability and maintenance concerns. Furthermore, as currently produced, these sensors are most useful for detecting when a nozzle drifts significantly in flow rate from other nozzles installed on the boom. This type of information is very useful for detecting plugged nozzles, but it does not address other sources of flow variations.

Various technologies exist that provide fluid flow measurements without requiring a moving element in the fluid. Sensors based on the propagation of ultrasonic waves are 
widely used in open channel flows (Muste et al., 2007), and some versions are also available for pipe flow systems (Sanderson and Yeung, 2002). However in ultrasonic-based sensors, the individual sensing elements are costly and require protection (Levesque and Oberg, 2012). This makes them less suitable for applications like individual nozzle monitoring, which requires a large number of individual sensing units. Laser Doppler anemometers (Adrian, 1983) and particle image velocimetry (Raffel et al., 2007) can also detect fluid flow rate and velocity without elements in the fluid flow, but these systems are generally expensive and limited to laboratory uses. Finally, electromagnetic flowmeters are also used for detecting fluid flow in pipes (Shercliff, 1962), but operation of these devices is difficult in low-conductivity fluids (White, 2003). Although some fluids used in agricultural sprayers could be highly conductive because of the salts in some agrochemicals, an electromagnetic flowmeter would be limited to applications of those chemicals.

The sensor technology developed in this study was designed to be able to determine the volumetric flow rate of an individual nozzle without any elements in direct contact with the fluid. It accomplishes this using LED and phototransistor pairs to detect the fluid's optical properties at two locations and then processing the signals using crosscorrelation. It is based on a water velocity sensor developed by Zhang et al. (2013), which likewise used crosscorrelation. Zhang et al. (2013) developed their sensor for determining open channel water velocity, but they also reported laboratory testing with enclosed pipe flow. Their sensor was based on an earlier sensor designed to determine the velocity of grain and other particles (Eamopas et al., 1994, 1997). All of these sensors were developed and tested for their effectiveness in determining the velocity of particles or fluids instead of the volumetric flow rate, which is the parameter of interest in this study. Finally, it is important to acknowledge that sensors with similar structures have also seen use in research in sprayer systems to measure properties other than flow rate. Vondricka and Lammers (2009) used a single LED and phototransistor pair to evaluate mixture homogeneity in direct injection sprayer applications.

The objective of this study was to evaluate the ability of correlation-based optical sensor technology to determine volumetric flow rate from a single nozzle as used on an agricultural sprayer system. In the previously cited studies, this optical cross-correlation technique was used only to monitor the velocity of various objects or fluids, rather than the volumetric flow rate. These studies also found situations where the technique might not be well suited. In Zhang et al. (2013), the mean percent error at certain velocities was as high as $11 \%$, and differences were noted in sensor operation above and below a velocity of $0.5 \mathrm{~m} \mathrm{~s}^{-1}$. Although these earlier studies established the applicability of an optical cross-correlation technique in certain circumstances, they did not provide any indication whether the technique would work inside a spray nozzle for determining flow rate. The fluid in a sprayer is pressurized, and the sensor is directly in front of a nozzle that causes enormous changes in flow characteristics. This is in contrast to the experiments of Zhang et al. (2013), which used low pressure and constant flow conditions.

This study sought to determine if the technique could be used successfully for determining the flow rates from nozzles as used in agricultural sprayers. Therefore, the testing focused on determining the baseline operation of this technology. The sprayer system was simulated using a small section of dry boom with a single sprayer nozzle attached. Testing included three extended-range flat spray nozzles operated at 100 to $400 \mathrm{kPa}$ to test flow rates from 0.46 to $2.74 \mathrm{~L} \mathrm{~min}^{-1}$. Since the testing focused on evaluation of the technique and not a particular sensor construction, the sensor signal processing used laboratory equipment rather than systems optimized for embedded operation or control of large numbers of sensors simultaneously. Future installation of this sensor design on sprayers would require replacement of the laboratory equipment with more suitable electronics. Pressure and flow rate were maintained at constant values during the tests. Complications such as pressure and flow fluctuations from boom section control and pulsations from PWM variable-rate nozzles were not considered to maintain the focus on baseline operation. Although these simplifications mean that the tests did not cover conditions experienced by the largest and most advanced sprayers, the test are still representative of the large number of basic sprayers that are currently operated at constant pressure and flow rate. Therefore, these tests should reveal the sensor's feasibility as a flow rate sensor technology for sprayers.

\section{Materials AND Methods}

\section{Sensor Technology and Operating Principle}

The sensor operates as shown in figure 1. This technology requires a sensor body with which the optical properties of the fluid flowing through it can be monitored. The optical properties must be observed at two locations. In this article, these locations are referred to as the upstream (closest to the

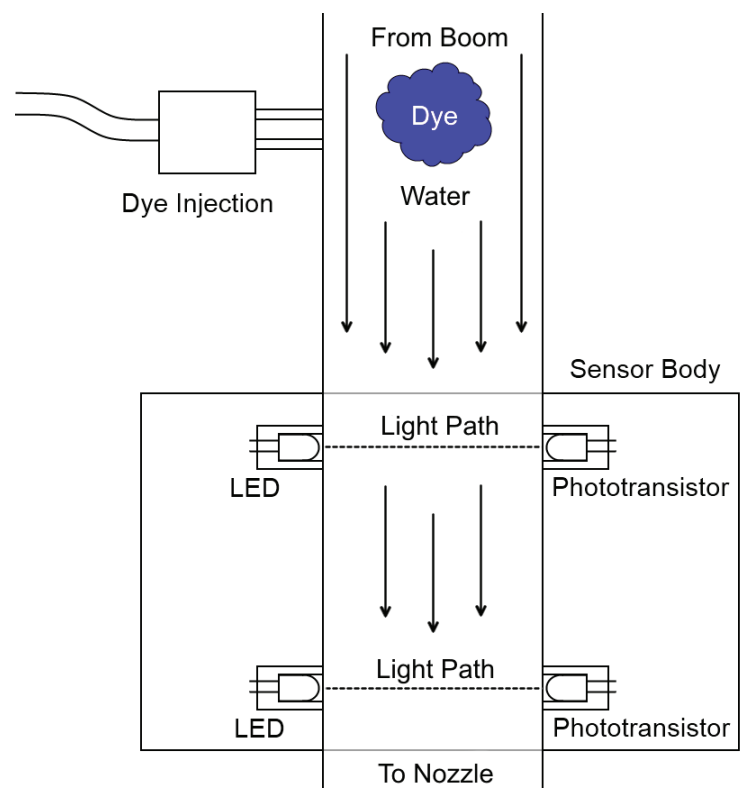

Figure 1. Illustration of sensor operating principle. 
boom) and downstream (closest to the nozzle) locations. The sensor also requires variability in the optical properties of the fluid. This variability can be intentionally introduced using a dye, as was done by Zhang et al. (2013). Natural variability in the fluid can also be sufficient for this technique under certain circumstances (Dvorak, 2012). However, using natural variability rather than induced variability from dye injection requires a fluid that blocks the transmission of a significant portion of the light and turbulent eddies to generate differences. Given the fact that sprayers are at times operated with transparent fluids, this testing focused on using induced variation from dye injection.

Cross-correlation is a statistical procedure that is used to determine the time difference between two signals (Bendat and Piersol, 1986, 1993). In this case, it was used to determine the time difference between when the dye affected the optical transmission properties at the upstream and the downstream locations. However, since these signals were not zero mean, the cross-covariance function rather than the standard cross-correlation function was more appropriate (Bendat and Piersol, 1993).There are multiple estimators for cross-covariance; in this experiment, the biased estimate of cross-covariance was used. The biased estimate of crosscovariance is:

$$
\hat{C}_{x y}(r T)=\frac{1}{N} \sum_{n=1}^{N-r}\left(x_{n}-\mu_{x}\right)\left(y_{n+r}-\mu_{y}\right)
$$

where

$\hat{C}_{x y}(r T)=$ discrete-time estimate of the cross-covariance

$x(t)$ and $y(t)=$ upstream and downstream signals, respectively (V)

$r=$ number of samples by which the downstream signal is shifted (also called lag number)

$T=$ sampling interval (s)

$N=$ total number of samples

$\mu_{x}=$ mean of $x(t)$

$\mu_{y}=$ mean of $y(t)$.

The cross-correlation coefficient scales the results of the cross-covariance function to between -1 and 1 (Bendat and Piersol, 1986). A value of 0 at a particular time delay indicates that the signals are uncorrelated at that delay. A value closer to 1 indicates a stronger correlation between the signals. A value closer to -1 indicates that one signal is more strongly correlated with the inverse of the other signal (Jenkins and Watts, 1968). The cross-correlation coefficient is calculated from the cross-covariance using:

$$
\rho_{x y}(\tau)=\frac{C_{x y}(\tau)}{\sigma_{x} \sigma_{y}}=\frac{C_{x y}(\tau)}{\sqrt{C_{x x}(0) C_{y y}(0)}}
$$

where

$C_{x y}(\tau)=$ cross-covariance

$\tau=$ time delay $(\mathrm{s})$

$\rho_{x y}(\tau)=$ cross-correlation coefficient

$x(t)$ and $y(t)=$ upstream and downstream signals, respectively (V)

$\sigma_{x}$ and $\sigma_{y}=$ standard deviations of $x(t)$ and $y(t)$, respectively.
After calculating the cross-correlation coefficient, the time delay between the signals is determined by finding the time delay at which the cross-correlation coefficient has its maximum value. A set of signals recorded in testing is shown in figure 2 , and the resulting cross-correlation coefficient from these signals is shown in figure 3 . The peak in the cross-correlation coefficient is highlighted in figure 3. These figures also demonstrate the robustness of the statistical approach to time delay determination provided by the cross-correlation coefficient. Even though the signals do not match exactly, the cross-correlation coefficient provided a strong signal indicating the time delay. Zhang et al. (2013) provide a more in-depth discussion of crosscovariance and the cross-correlation coefficient.

The time delay that results from the cross-correlation calculation should be related to the flow rate from any nozzle that is connected downstream from the sensor. However, this relationship is complicated by the fact that fluid flow velocity is not constant, and even the velocity profiles within the pipe vary as flow regimes change from laminar to turbulent flow. Other effects, such as diffusion of the

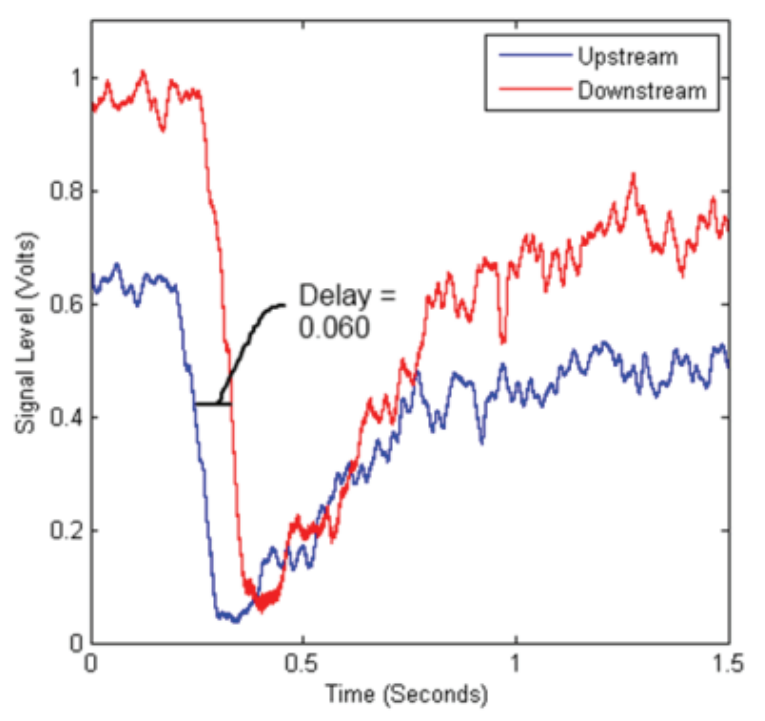

Figure 2. Example phototransistor signals.

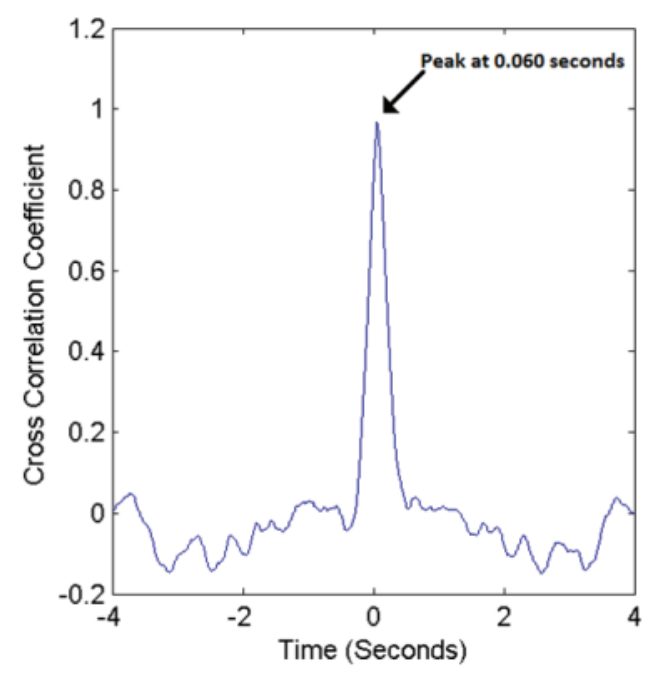

Figure 3. Cross-correlation coefficient of the signals in figure 2. 
dye, create even more complications in the relationship. Zhang et al. (2013) discuss and show the results of some of these influences. Rather than trying to account for every single factor of fluid dynamics that could affect the way the dye flows between the sensing locations, this testing focuses on determining the flow rate from a nozzle directly from the time delay measured by this sensor technique.

\section{SENSOR TECHNOLOGY IMPLEMENTATION FOR EXPERIMENT}

Testing of this sensing technique required a basic implementation of this system. This necessitated the construction of a dye injection system, a sensor body through which the fluid flowed, and the electrical components necessary to monitor the optical properties of the fluid at two locations. The system developed for this testing should not be considered a final sensor design, or even a prototype of a final sensor design. This system was created for proof-ofconcept testing, and the selected components were used based on their suitability for laboratory testing.

A small amount of dye was injected before the flow entered the sensor body in order to create a difference in the optical properties of the flow through the sensor. This dye was Standard Blue Dye from Bright Dyes (Miamisburg, Ohio), which is a special formulation of Acid Blue 9. It was used at a concentration of $5 \mathrm{~g}$ of dry powder per liter. This dye is sold for use in outdoor bodies of water (Bright Dyes, 2014). This dye has the same primary ingredient as the dye used by Zhang et al. (2013). An excellent review of its toxicology, possible environmental effects, and suitability as a tracer dye is given by Flury and Flühler $(1994,1995)$. The dye was stored in a container at $690 \mathrm{kPa}$ and was injected into the water by activating a solenoid (71216SN2BL00N0C111C2, Parker, New Britain, Conn.) connected to a tee fitting installed directly before the main sensor body. An activation period of $15 \mathrm{~ms}$ was selected for the solenoid, as this was the minimum (to limit dye usage) at which the dye was consistently injected at all boom pressures tested. Although there are more exact metering methods and injection techniques, this simple system was used because the final objective of the project was to establish if the optical cross-correlation technique could be useful in determining the flow rate of individual nozzles. To be practical, any future design would also require a simple dye injection system, which disqualifies a more complicated and expensive injection system. Given that more advanced methods would be impractical in a final design, this initial testing used the simple injection system presented here.

The sensing body for testing was connected directly before the nozzle, between the nozzle and the boom. It was constructed of solid aluminum into which were mounted two sets of LEDs and phototransistors. These devices were securely installed and water-sealed with transparent potting compound (EP965 Clear, Resinlab, Germantown, Wisc.). The sensor structure used for testing is shown in figure 4. The internal diameter of the flow path through the sensor was $6.35 \mathrm{~mm}$ to match the internal diameter of the $1 / 4$ inch NPT fittings used on the nozzle and boom. The sensor body was connected to the nozzle and boom using 1/4 inch NPT threads tapped into the sensor body.
The electrical circuit for operating the LEDs and phototransistors and for signal conditioning is shown in figure 5. The LEDs (C503B-RAS-CY0B0AA1, Cree, Inc., Durham, N.C.) are D1 and D2 in figure 5, and the phototransistors (SFH314, Osram Opto Semiconductors GmbH, Regensburg, Germany) are Q1 and Q2. Potentiometers (10 k $\Omega)$, R5 and R6 in figure 5, were used to convert the current output from the phototransistors into a voltage signal. The potentiometers were adjusted so that the output voltage was greater than $0.5 \mathrm{~V}$ when clear water was flowing through the sensor body but less than the $3.3 \mathrm{~V}$ power supply used. The signal processing method (cross-correlation) was not affected by the magnitude of the inputs as long as it could be adequately captured by the processing hardware, since the cross-correlation was only responding to the time delay between the signals. These signals were then buffered by voltage follower circuits using operational amplifiers (OPA4344, Texas Instruments, Dallas, Tex.).

The signals were recorded at $50 \mathrm{kHz}$ using a data acquisition board (USB-6259, National Instruments, Austin, Tex.). The high sample rate for signal acquisition recorded extra high-frequency noise and created large data sets, which increased processing time. However, the statistical nature of the cross-correlation calculation meant that uncorrelated noise did not affect the time delay determination, while noise correlated with the flow through the sensor would increase its ability to detect the time delay. Higher sample rates also decrease the discretization error of the time delay estimation. Therefore, at a cost of increased calculation time, the signals were recorded at a high sample rate. After ascertaining the ability of this type of sensor to determine nozzle flow rate, future work can investigate the minimum sample rate necessary to accurately determine the time delay so that processing time can be minimized, which will be important for future applications on sprayers in the field.

\section{EXPERIMENTAL SETUP}

The experiments were conducted on a small section of dry boom on a spray table. Only one nozzle was installed on the boom during tests. Water flow was provided by an electrically powered pump (101BM07MC, Oberdorfer, Syracuse, N.Y.), and pressure was set using a pressure relief valve (model 23120, Spraying Systems Co., Wheaton, Ill.). The water flow was carried to the nozzle by flexible hose with an internal diameter of $19.05 \mathrm{~mm}$. The water was municipal tap water (Lexington, Ky.) at a temperature of $20^{\circ} \mathrm{C}$. Water bypassed by the pressure relief valve was recirculated to the holding tank, but all water that flowed through the boom was not recirculated in this testing. All connections were made using fittings that were $1 / 4$ inch NPT style. The nozzle was attached using Teejet's $1 / 4$ inch NPT to Quick Teejet Adaptor (QJ1/4TT-NYB, Spraying Systems Co., Wheaton, Ill.). The experimental setup is shown in figure 6.

During the experiment, three different nozzles and four different pressures were used. For each combination of nozzle and pressure, 35 separate measurements of flow rate were made using the system. Five of these samples were 

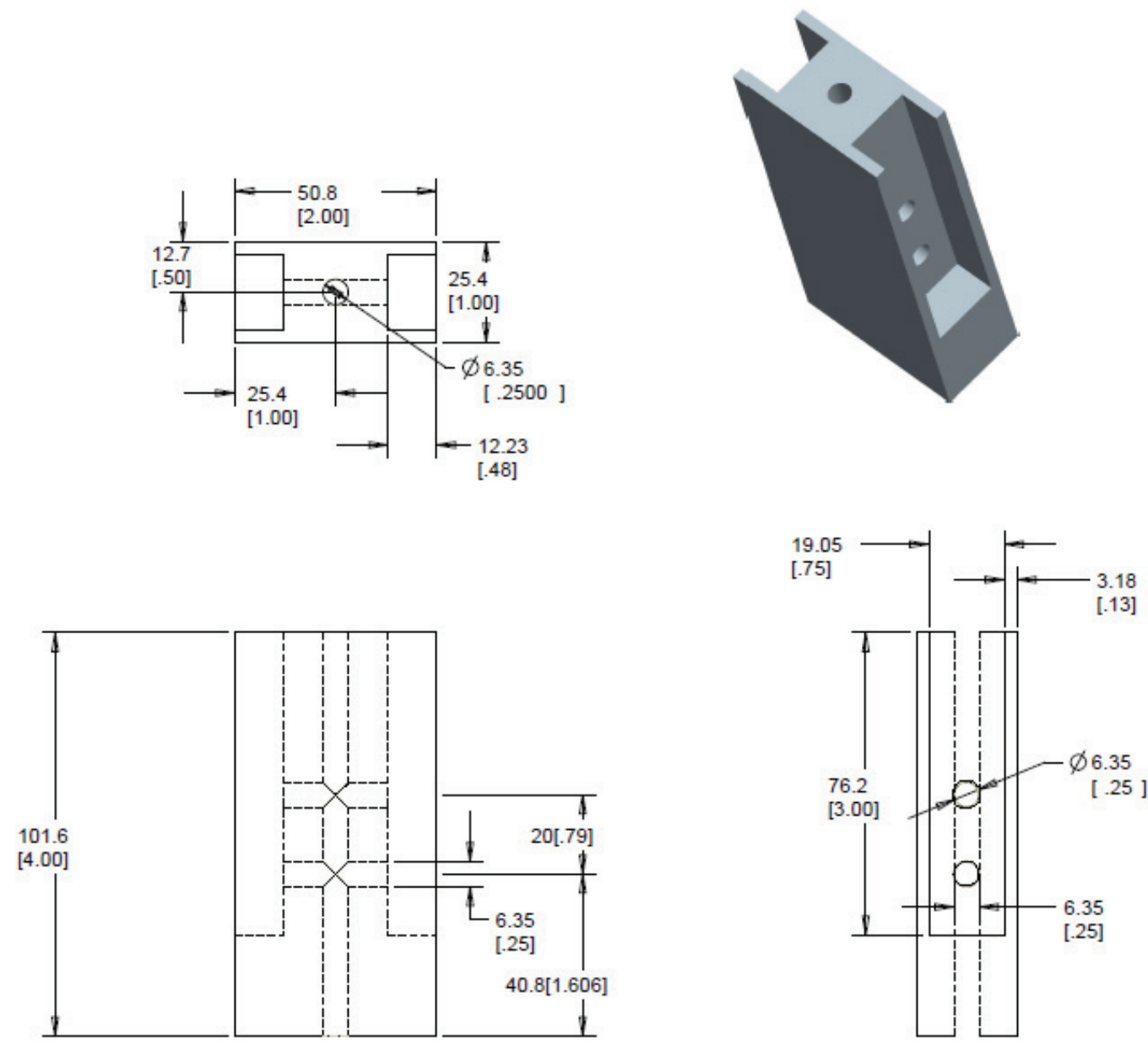

Figure 4. Sensor body design drawing. Dimensions are mm [inches].

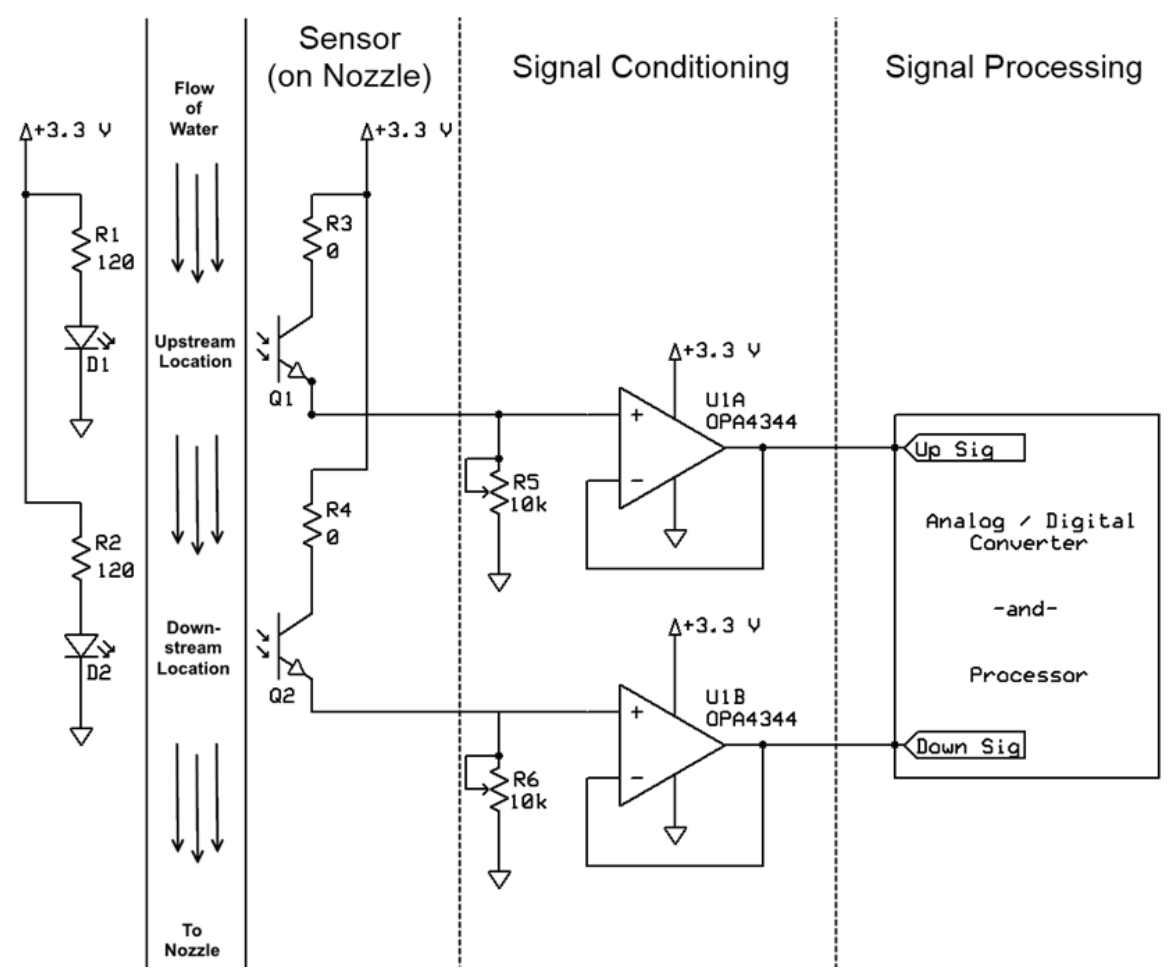

Figure 5. Schematic of electrical components in the sensor and the signal conditioning circuits. 


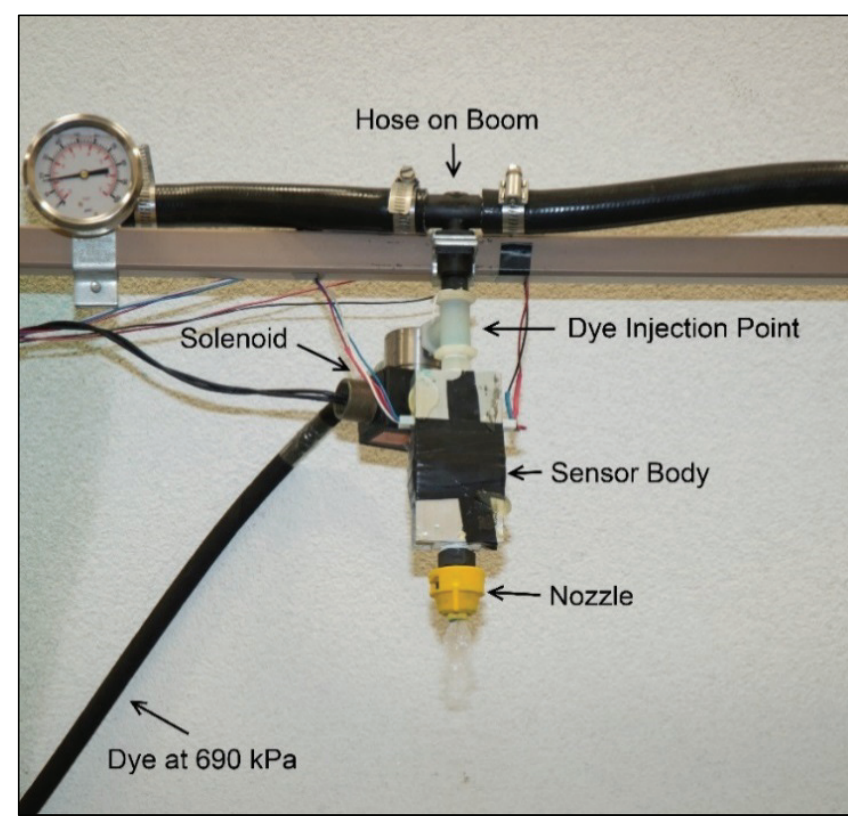

Figure 6. Experimental setup.

randomly selected to produce the calibration curve. The remaining 30 samples were used for validation. As each flow rate measurement using the optical cross-correlation technique was being made, a verification measurement was made using a graduated cylinder and a stopwatch to determine the actual flow rate, which is also called "true flow" in the Results and Discussion section. The three nozzles tested were the XR8002VS, XRC8004, and XRC8006, all from Teejet Technologies (Spraying Systems Co., Wheaton, Ill.). These are basic $80^{\circ}$ extended-range flat spray nozzles. The tested pressures were 100, 200, 300, and $400 \mathrm{kPa}$. Nominal expected flow rates in this experiment ranged from 0.46 to $2.74 \mathrm{~L} \mathrm{~min}^{-1}$. Droplet size varied from fine to coarse. Reynolds numbers through the sensor body varied from 1500 to 9300 , which corresponds to flow regimes from laminar through transitional to fully turbulent. Table 1 lists the flow conditions in each test. Both the flow rates that were expected based on catalog specifications and the flow rates that were actually measured in testing are listed in this table, as there were slight variations.

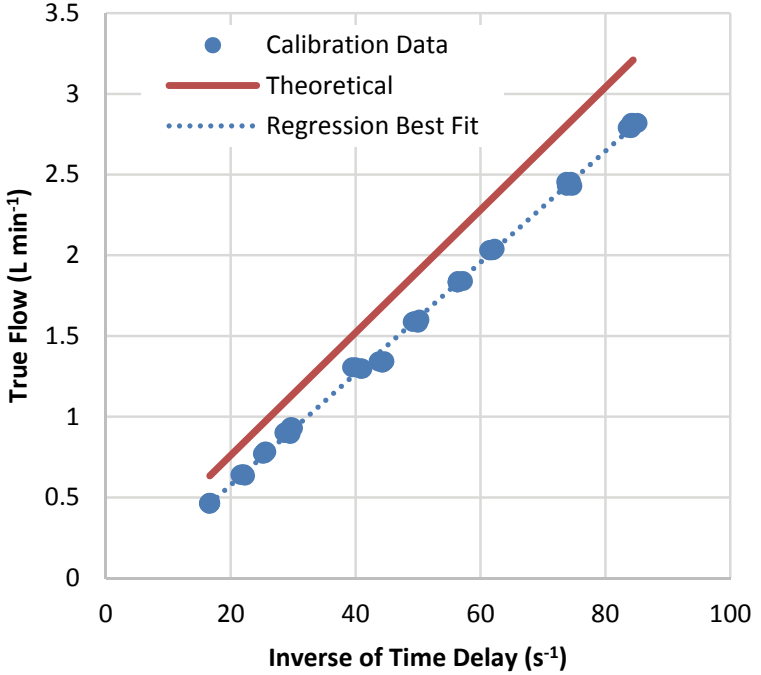

Figure 7. Plot of the 60 calibration points showing the relationship between the sensor output (expressed as inverse of time delay) and the measured flow rate. The theoretical relationship discussed in the text is shown for comparison.

\section{RESULTS AND DISCUSSION}

The property of interest in this study was the flow rate from the nozzle, but the sensing technique's output was actually the travel time for the dye. This travel time was expressed as the time delay between the two signals. Therefore, a calibration was necessary to relate time delay to flow rate. Since the equations for velocity and flow rate indicate that flow rate should be linear with the inverse of the time delay, the calibration was performed with the inverse of time delay and the flow rate. A plot of the calibration points is shown in figure 7. As the relationship appeared linear, a linear regression was performed, and the resulting equation for the relationship was $Q=0.0345\left(1 / t_{d}\right)$ -0.1128 , where $Q$ is flow rate in $\mathrm{L} \mathrm{min}^{-1}$, and $t_{d}$ is time delay in $\mathrm{s}$. The $\mathrm{R}^{2}$ value from the regression was 0.9986 .

Interestingly, this equation is not the one that would be predicted based solely on velocity and flow rate equations if we assumed that the measured time delay is directly related to the average velocity. With a diameter of $6.35 \mathrm{~mm}$ for the flow path and $20 \mathrm{~mm}$ separation between the two LED/phototransistor locations, the equation based on the

Table 1. Flow properties during each test.

\begin{tabular}{|c|c|c|c|c|c|c|}
\hline $\begin{array}{c}\text { Nozzle } \\
\text { Part Number }\end{array}$ & $\begin{array}{c}\text { Pressure } \\
(\mathrm{kPa})\end{array}$ & $\begin{array}{c}\text { Expected Discharge }{ }^{[\mathrm{a}]} \\
\left(\mathrm{L} \mathrm{min}^{-1}\right)\end{array}$ & $\begin{array}{c}\text { Measured Discharge } \\
\left(\mathrm{L} \mathrm{min}^{-1}\right)\end{array}$ & $\begin{array}{l}\text { Reynolds } \\
\text { Number }^{[\mathrm{b}]}\end{array}$ & $\begin{array}{c}\text { Flow } \\
\text { Regime }\end{array}$ & $\begin{array}{c}\text { Expected Droplet } \\
\text { Size }^{[\mathrm{a}]}\end{array}$ \\
\hline \multirow{4}{*}{ XR8002VS } & 100 & 0.46 & 0.46 & 1500 & Laminar & Medium \\
\hline & 200 & 0.65 & 0.64 & 2100 & Laminar & Medium \\
\hline & 300 & 0.79 & 0.77 & 2600 & Transitional & Fine \\
\hline & 400 & 0.91 & 0.90 & 3000 & Transitional & Fine \\
\hline \multirow{4}{*}{ XRC8004 } & 100 & 0.91 & 0.93 & 3100 & Transitional & Coarse \\
\hline & 200 & 1.29 & 1.30 & 4300 & Turbulent & Medium \\
\hline & 300 & 1.58 & 1.59 & 5200 & Turbulent & Medium \\
\hline & 400 & 1.82 & 1.83 & 6100 & Turbulent & Medium \\
\hline \multirow{4}{*}{ XRC8006 } & 100 & 1.37 & 1.33 & 4400 & Turbulent & Coarse \\
\hline & 200 & 1.94 & 2.03 & 6700 & Turbulent & Coarse \\
\hline & 300 & 2.37 & 2.44 & 8000 & Turbulent & Coarse \\
\hline & 400 & 2.74 & 2.81 & 9300 & Turbulent & Coarse \\
\hline
\end{tabular}

[a] From Teejet catalog (Spraying Systems Co., Wheaton, Ill.).

[b] For flow through the sensor. 


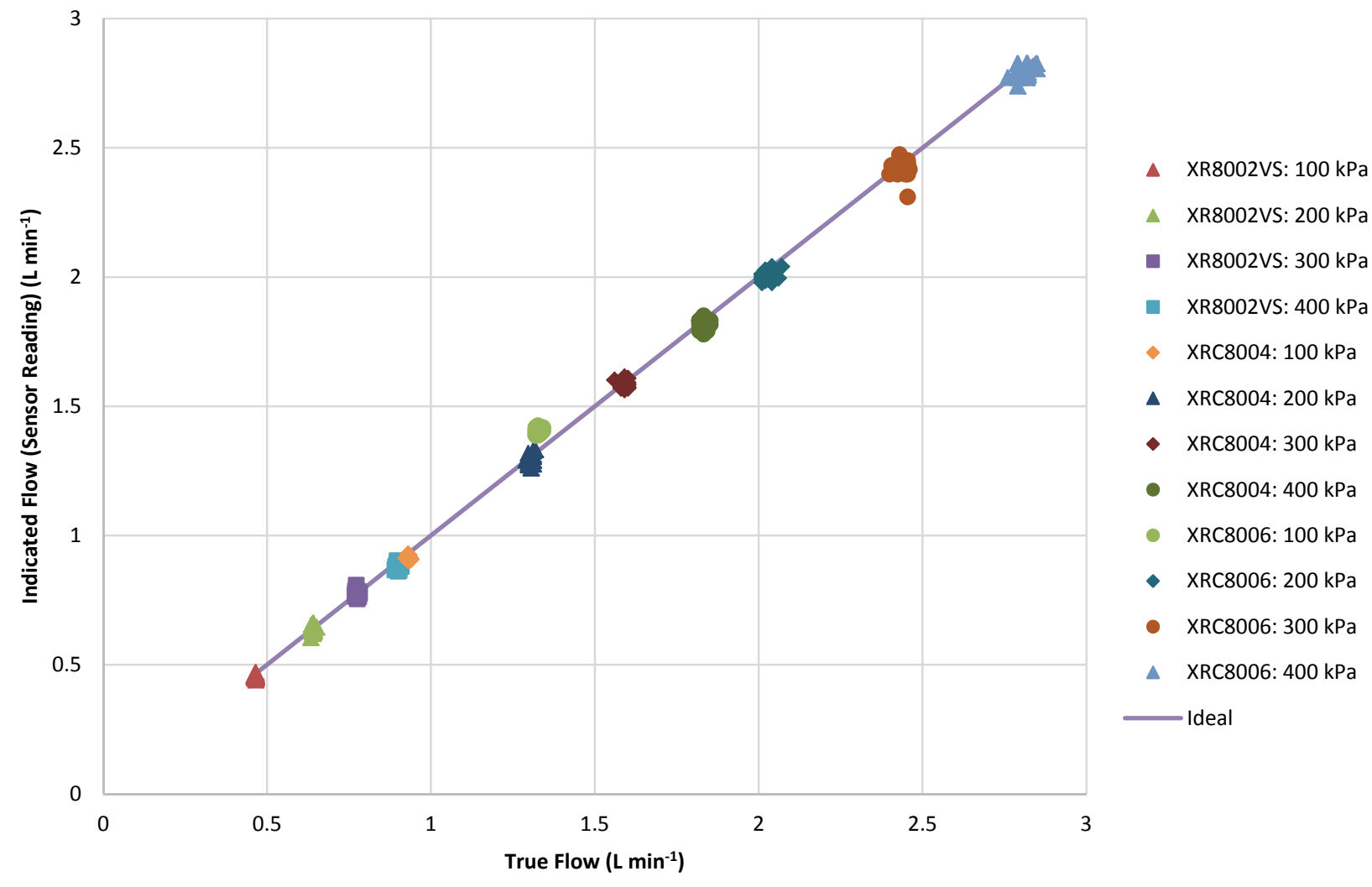

Figure 8. Results from the validation test of the sensor.

proceeding assumptions would be $Q=0.038\left(1 / t_{d}\right)$, with the same units and notation as before. However, these assumptions do not take into account the velocity profile variations and fluid/dye mixing differences that occur as the Reynolds number and flow regime change across the flow rates expected in an agricultural spraying operation. These phenomena have an effect, as the equations are not the same. However, based on the strong linearity of the calibration relationship, any non-linear contributions from these phenomena are insignificant within the flow rates and system parameters expected in agricultural sprayers.

For validation, the indicated flow determined using the calibration equation was compared to the true flow using 30 measurements at each nozzle/pressure combination. The results from this validation test are shown in figure 8 . The ideal relationship, i.e., indicated flow $=$ true flow, is shown as a solid line in figure 8 for comparison. Upon visually inspecting figure 8 , it can be seen that all the measurements are clustered around the ideal relationship except for those from the XRC8006 nozzle at $100 \mathrm{kPa}$. A linear regression was performed on the validation measurements, and the results are shown in table 2 . The linear regression confirmed that the best fit line was very close to the ideal, indicated flow $=$ true flow (where the slope is 1 and the inter-

Table 2. Results from linear regression of validation data.

\begin{tabular}{cccc}
\hline $\begin{array}{c}\text { Regression Best Fit } \\
\text { Equation }\end{array}$ & \multicolumn{2}{c}{$95 \%$ Confidence Interval for: } \\
\cline { 2 - 4 } & $\mathrm{R}^{2}$ & Slope & Intercept \\
\hline$Q_{p}=Q_{m} \times 0.996-0.002$ & 0.998 & 0.991 to 1.000 & -0.005 to 0.009 \\
\hline [a] $Q_{p}=$ indicated flow $\left(\mathrm{L} \mathrm{min}^{-1}\right)$, and $Q_{m}=$ true flow $\left(\mathrm{L} \mathrm{min}^{-1}\right)$.
\end{tabular}

cept is 0 ). The $95 \%$ confidence intervals for the slope and intercept both include their ideal values.

The residuals from the regression analysis are shown in figure 9. The cluster of points with residuals near $0.1 \mathrm{~L}$ $\mathrm{min}^{-1}$ at a true flow rate of $1.3 \mathrm{~L} \mathrm{~min}^{-1}$ all come from the XRC8006 nozzle at $100 \mathrm{kPa}$. The residuals for the other test settings are clustered around 0 . The residual plot also reveals that the measurement error is not constant across the entire range but appears to increase as the reading increases. While the error increases with flow, the percent error of the readings (fig. 10) decreases slightly as flow increases. Once again, in figure 10 , the test with the XRC8006 nozzle at $100 \mathrm{kPa}$ stands out from the rest, with percent errors clustered around 6\%. Table 3 summarizes

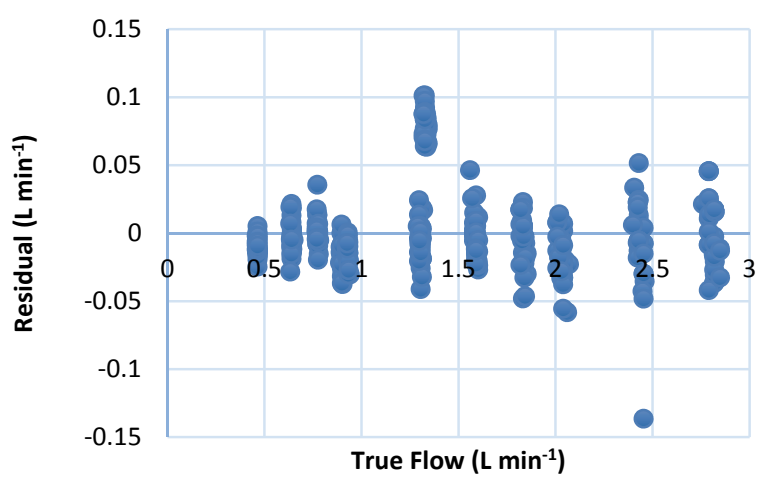

Figure 9. Residuals from regression of data from the validation test. 


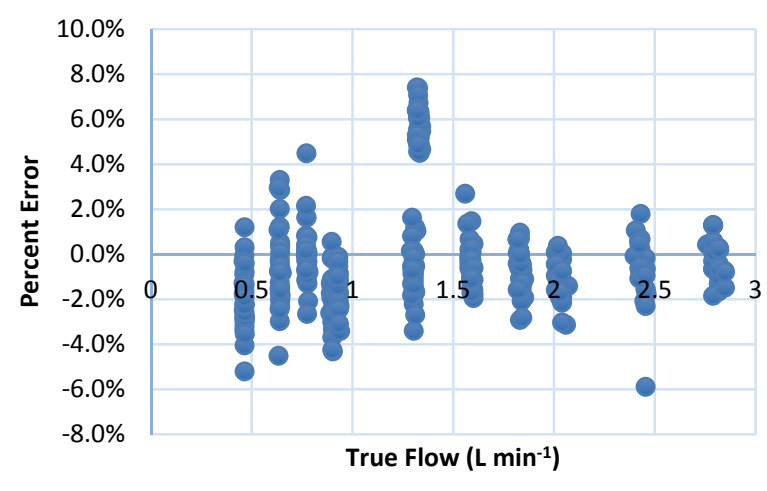

Figure 10. Percent error of the readings in the validation test.

Table 3. Error of sensor based on the validation data.

\begin{tabular}{cccccc}
\hline & \multicolumn{2}{c}{$\begin{array}{c}\text { Absolute Percent } \\
\text { Error }(\%)\end{array}$} & & \multicolumn{2}{c}{$\begin{array}{c}\text { Absolute Error } \\
\left(\mathrm{L} \mathrm{min} \text { m }^{-1}\right)\end{array}$} \\
\cline { 2 - 3 } \cline { 5 - 6 } & Worst & Mean & & Worst & Mean \\
\hline All data & 7.4 & 1.6 & & 0.136 & 0.020 \\
\hline $\begin{array}{c}\text { Without XRC8006 } \\
\text { nozzle at 100 kPa }\end{array}$ & 5.9 & 1.3 & & 0.136 & 0.014 \\
\hline
\end{tabular}

the error of the readings from the validation data. The table also shows the same results without the readings taken with the XRC8006 nozzle at $100 \mathrm{kPa}$, since the error of those readings appeared clearly different from the other readings. However, including those readings did not change the worst absolute error and only increased the worst absolute percent error by $1.5 \%$.

The measurements obtained using the XRC8006 nozzle at $100 \mathrm{kPa}$ are a concern, as they do not cluster on the ideal relationship line. This discrepancy cannot be related to the Reynolds number of the flow through the sensor, as the Reynolds number of the flow through the sensor for the XRC8006 nozzle at $100 \mathrm{kPa}$ is nearly the same as that for the XRC8004 nozzle at $200 \mathrm{kPa}$. This discrepancy is also not a direct effect of the pressure, the nozzle, or the flow rate, as other nozzles did not have this issue at $100 \mathrm{kPa}$, the XRC8006 nozzle did not have this issue at other pressures, and the flow rate is in the middle of those tested. The difference is likely related to the flow characteristics through the nozzle (and not those through the sensor), which directly affect the observable spray characteristics such as droplet size. The droplet size was rated as coarse for the XRC8006 nozzle at $100 \mathrm{kPa}$ (Teejet, 2011). The droplet size for the other test conditions ranged from fine to coarse, but the XRC8006 at $100 \mathrm{kPa}$ produced the coarsest droplets of all test conditions, as this test setup had the highest capacity nozzle at the lowest pressure. The results from the XRC8006 nozzle at $100 \mathrm{kPa}$ indicate that care must be taken when using this flow rate measuring approach with nozzles and conditions that produce coarse or larger droplet sizes. In these situations, it is likely that the flow characteristics are different enough that a simply linear relationship between flow rate and dye travel time does not hold. Tests will need to be performed with each nozzle type with which this sensor is used to make sure the assumption of a linear calibration remains true. Future work will involve testing with different classes of nozzles producing different droplet sizes to determine if different linear calibration curves are necessary for different nozzles. Other testing will need to be performed to determine how droplet size affects this sensing technology.

\section{CONCLUSION}

This article presents the design of a robust, optical crosscorrelation based sensor technology for sprayer nozzle flow rate, the data processing and calibration necessary to determine the flow rate, and validation of the technique at a variety of common sprayer pressures and flow rates. The tests presented in this article show that an optical crosscorrelation based sensor can determine nozzle flow rate in an agricultural sprayer system. The worst absolute error in percent was $7.9 \%$, while the mean absolute error in percent was $1.6 \%$ for all 360 measurements taken at various pressures and with different nozzles. One strength of this sensor technology is its robustness. The sensor body contains no moving parts and does not affect the flow any more than would the addition of a short section of tube. This could reduce issues such as jamming, plugging, or wearing out of sensor components. The biggest drawback of the sensor technique is the requirement for a tracer dye. Tracer dyes have long been used on sprayers to mark coverage paths in commercial applications and to measure spray deposition in research. In addition, researchers are actively seeking improvements in tracer dyes (Hoffmann et al., 2014). However, the requirement for dye injection adds components and complexity to a sprayer, which could be undesirable. This may not be a big drawback if this sensor system were combined with other systems that also use a tracer dye, such as direct injection concentration measurements.

Although this system worked well with one common spray nozzle type and at a range of common flow rates and pressures, further testing will be necessary to ascertain its applicability for a much wider range of spray nozzle types and sprayer conditions. In addition to testing other nozzle types and spray droplet sizes, these tests need to include common spray control techniques, such as PWM control. It is also important to determine if the dye injection can occur at a central location on the boom instead of at each individual nozzle, as this will make installation of the entire system much simpler. In this case, dye would be injected centrally, and the sensors on the individual nozzles would observe the effect of the dye when it reached that nozzle. Further testing is necessary before this system can be suggested as an alternative to the nozzle flow rate sensors currently used on agricultural systems. However, the tests presented here confirm that this technology can work well as a method of determining the flow rate in individual nozzles on an agricultural sprayer.

\section{REFERENCES}

Adrian, R. J. (1983). Laser velocimetry. In (R. J. Goldstein, ed.) Fluid Mechanics Measurement (pp. 155-244) Washington, D.C.: Hemisphere Publishing.

Bendat, J. S., \& Piersol, A. G. (1986). Random Data. New York, 
N.Y.: John Wiley and Sons.

Bendat, J. S., \& Piersol, A. G. (1993). Engineering Applications of Correlation and Spectral Analysis. New York, N.Y.: John Wiley and Sons.

Bright Dyes. (2014). FAQs. Miamisburg, Ohio: Bright Dyes, A Division of Kingscote Chemicals. Retrieved from www.brightdyes.com/FAQ.html.

Dvorak, J. S. (2012). An optical water velocity sensor for open channel flows. PhD diss. Manhattan, Kans.: Kansas State University.

Eamopas, K., Zhang, N., \& Schrock, M. D. (1994). Monitoring the glass bead dispensing rate for highway striping vehicles. ITE J., 64(4), 41-46. Washington, D.C.: Institute of Transportation Engineers.

Eamopas, K., Zhang, N., \& Schrock, M. D. (1997). Parameter selection for correlation-based particle velocity sensors. Trans. ASAE, 40(5), 1501-1508.

http://dx.doi.org/10.13031/2013.21372.

Flury, M., \& Flühler, H. (1994). Brilliant blue FCF as a dye tracer for solute transport studies: A toxicological overview. $J$. Environ. Qual., 23(5), 1108-1112. http://dx.doi.org/10.2134/jeq1994.00472425002300050037x.

Flury, M., \& Flühler, H. (1995). Tracer characteristics of brilliant blue FCF. SSSA J., 59(1), 22-27. http://dx.doi.org/10.2136/sssaj1995.03615995005900010003x.

Grisso, R. D., Dickey, E. C., \& Schulze, L. D. (1989). The cost of misapplication of herbicides. Appl. Eng. Agric., 5(3), 344-347. http://dx.doi.org/10.13031/2013.26525.

Hoffmann, W. C., Fritz, B. K., \& Ledebuhr, M. A. (2014). Evaluation of 1,3,6,8-pyrene tetra sulfonic acid tetra sodium salt (PTSA) as an agricultural spray tracer dye. Appl. Eng. Agric., 30(1), 25-28. http://dx.doi.org/10.13031/aea.30.10313.

Hofman, V., \& Solseng, E. (1986). Spray equipment and calibration. Fargo, N.D.: North Dakota State University Cooperative Extension Service.

Huyghebaert, B., Debouche, C., \& Mostade, O. (2001). Flow rate quality of new flat fan nozzles. Trans. ASAE, 44(4), 769-773. http://dx.doi.org/10.13031/2013.6238.

Jenkins, G. M., \& Watts, D. G. (1968). Spectral Analysis and its Applications. San Francisco, Cal.: Holden-Day.

Levesque, V. A., \& Oberg, K. A. (2012). Computing discharge using the index velocity method. Techniques and Methods 3A23. Reston, Va.: U.S. Geological Survey.

Menzies, D. R., Fisher, R. W., \& Neff, A. E. (1976). Wear of hollow cone nozzles by suspensions of wettable powders. Canadian Agric. Eng., 18(1), 14-15.

Muste, M., Vermeyen, T., Hotchkiss, R., \& Oberg, K. (2007). Acoustic velocimetry for riverine environments. J. Hydraul. Eng., 133(12), 1297-1298. http://dx.doi.org/10.1061/ (ASCE)0733-9429(2007)133:12(1297).

Ozkan, H. E., Reichard, D. L., \& Ackerman, K. D. (1992). Effect of orifice wear on spray patterns from fan nozzles. Trans. ASAE, 35(4), 1091-1096. http://dx.doi.org/10.13031/2013.28704.

Raffel, M., Willert, C. E., Wereley, S. T., \& Kompenhans, J. (2007). Particle Image Velocimetry: A Practical Guide. New York, N.Y.: Springer.

Reichard, D. L., Ozkan, H. E., \& Fox, R. D. (1991). Nozzle wear rates and test procedures. Trans. ASAE, 34(6), 2309-2316. http://dx.doi.org/10.13031/2013.31873.

Sanderson, M. L., \& Yeung, H. (2002). Guidelines for the use of ultrasonic non-invasive metering techniques. Flow Measurement Instruments, 13(4), 125-142. http://dx.doi.org/10.1016/S0955-5986(02)00043-2.

Sharda, A., Fulton, J. P., McDonald, T. P., Zech, W. C., Darr, M. J., \& Brodbeck, C. J. (2010). Real-time pressure and flow dynamics due to boom section and individual nozzle control on agricultural sprayers. Trans. ASABE, 53(5), 1363-1371. http://dx.doi.org/10.13031/2013.34891.

Shercliff, J. A. (1962). The Theory of Electromagnetic FlowMeasurement. Cambridge, U.K.: Cambridge University Press.

Teejet. (2011). Teejet Technologies: Catalog 51-M. Wheaton, Ill.: Spraying Systems Co. Retrieved from www.teejet.com/media/409041/cat51-m_metric_lores_all.pdf.

Varner, D. L., Grisso, R. D., \& Shearman, R. C. (1990). Calibration accuracy of golf course pesticide applicators. Appl. Eng. Agric., 6(4), 405-411. http://dx.doi.org/10.13031/2013.26405.

Vondricka, J., \& Lammers, P. S. (2009). Measurement of mixture homogeneity in direct injection systems. Trans. ASABE, 52(1), 61-66. http://dx.doi.org/10.13031/2013.25941.

Wesley, M. P., Jorge, R., Yeyin, S., Randal, K. T., \& Paul, W. (2013). Laboratory evaluation of a turn compensation control system for a ground sprayer. Appl. Eng. Agric., 29(5), 655-662. http://dx.doi.org/10.13031/aea.29.10075.

White, F. M. (2003). Fluid Mechanics. New York, N.Y.: McGrawHill Higher Education.

Zhang, N., Dvorak, J. S., \& Zhang, Y. (2013). A correlation-based optical flowmeter for enclosed flows. Trans. ASABE, 56(6), 1511-1522. http://dx.doi.org/10.13031/trans.56.10052. 